\title{
Iterative Decoding of Chase Pyndiah Decoder Utilizing Multiple Relays Network
}

\author{
Saif E. A. Alnawayseh \\ Department of Electric Engineering \\ Faculty of Engineering, Mutah University \\ Karak, Jordan
}

\begin{abstract}
In this paper, a distributed Encoding and decoding of Turbo Product Code (TPC) over single and multiple relays network are proposed. The information message matrix is encoded at source by Bose Chaudhuri Hochquenghem (BCH) as component code and transmitted to destination and relays in the midway between source and destination. The coded source message is decoded by simple chase $\Pi$ decoder at relay and encoded again horizontally and vertically by BCH component code to construct TPC. Two scenarios were investigated. First scenario, utilizing one relay in cooperative network, where the vertical parity part of TPC is transmitted to destination to be the input of row decoder for original chase pyndiah decoder, while the received encoded horizontally matrix from source is the input of the column decoder. In the second scenario multiple relays are utilized and multiple copies of vertical parity part of TPC are sent to destination to be decoded by the proposed modified iterative chase pyndiah decoder with multiple integrated stages for each iteration. Simulation results for the first scenario over Additive White Gaussian (AWGN) and Rayleigh fading channels and using original chase Pyndiah decoder at destination shows $2 \mathrm{~dB}$ gain improvement at $\mathrm{BER}=10^{-5}$ and $4 \mathrm{~dB}$ gain improvement at $B E R=10^{-4}$ respectively over $B E R$ performance of un cooperative system. While results for distributed TPC decoding for the second scenario and using the proposed modified iterative chase Pyndiah decoder at destination shows $2.7 \mathrm{~dB}$ and $3 \mathrm{~dB}$ gain at $\mathrm{BER}=10^{-4}$ for AWGN and Rayleigh fading channels respectively over the first scenario.
\end{abstract}

Keywords-Turbo product code (TPC); modified iterative chase Pyndiah decoding algorithm; relay; source; Bit Error Rate (BER); vertical parities; horizontal parities

\section{INTRODUCTION}

Distributed decoding of turbo codes has been investigated widely over cooperative relay network system [1], [2], [3], [4], and [5]. One of the turbo codes types is the Turbo Product Code TPC. TPC is considered to be an efficient coding algorithm compared to convolutional code turbo coed [6] which has been adopted by different communication standards such as IEEE802.20 and IEEE802.16 [7]. Many characteristics of TPC lead it to be one of the main coding schemes such as using simple Soft Input Soft Output (SISO) decoding algorithms that can reach near Shannon capacity limit by acceptable level of complexity of decoding especially at high code rates [8] such as iterative Pyndiah decoder [9] and many other decoding algorithms as in [10]. Moreover, TPC is easily constructed by components code which gives flexibility and high degree of parallelized structure and simple encoding. This simplicity and flexibility in encoding and decoding process can be employed widely in coded cooperative relay communication system. Such system has gained a lot of interest recently as the diversity it provides against fading for wireless networks [11] and [12]. Furthermore, relay cooperative system is a good diversity alternative for multiple antennas where the size is a matter especially for wireless sensor networks [11]. The main idea of this system is based on the fact that inter user channel between source and relay or relay and destination are assumed to be more reliable than direct link between source and destination.

Many cooperative techniques are implemented according to this idea such as amplify and forward (AF), Decode and Forward (DF), and coded cooperation [13], [14], [15], and [16]. In this field coded cooperative using TPC shows a high performance with low complexity compared with conventional distributed codes [17] for its powerful BER performance and high rate code. Many works and ideas have been investigated to utilize TPC in single or multiple relays network. In [17] and [18] distributed TPC was investigated by sending BCH code to relays and destination. Where the received sequences at relays are decoded then encoded horizontally and interleaved circularly and encoded vertically to produce multiple copies of vertical parities that are send to destination to be decoded by modified SISO Turbo decoder. For maximum transfer information from relay a soft parity generating method that produces soft incremental redundancy was proposed in [19] using chase $\Pi$ soft decoding algorithm and distance based decoding. A further improvement for distributed TPC was presented in [20] using power allocation technique rather than assigning equally power to source and relay as done in [17] and [19]. Such method reduced the effect of relay decoding error compared to distribute TPC with fixed power assignment.

In this paper a distributed TPC is proposed by applying the idea of distributed encoding and decoding over single and multiple relay networks. Where the source message is distributed encoded between sources and relays to produce TPC, and distributed decoding over multiple channels between relays and destination utilizing modified iterative chase pyndiah decoder for TPC at destination. For efficient data transmission between relays and destination the source coded message matrix is decoded by chase $\|$ algorithm at relay and reencoded gain horizontally and vertically using $\mathrm{BCH}$ component codes. The vertically parity encoded matrix is sent to destination to be the input of row decoder while the encoded message matrix from source is applied to the column decoder of iterative chase pyndiah decoder. For the case of multiple 
relays, the modified iterative chase pyndiah will contain integrated multiple stages for each half iteration that capable to receive multiple versions of vertical parity matrixes from multiple relays. The main difference in the modified chase pyndiah decoder in this work compared to previous work in literature such as in [17] is that the extrinsic of the column decoder $w_{i c}{ }^{\prime}$ in the first sub stage will be the input extrinsic for the next row decoder in the second sub stage. Also the Extrinsic of row decoder of second sub stage $w_{i h}^{\prime}$ will be the input for columns decoder. As a result, more efficient utilization for iterations in chase pyndiah iterative decoding as will be explained in details in next sections.

\section{SYSTEM MODEL}

We consider BCH systematic linear block codes $C_{1} \cdot C_{2}$ with parameters $\left(N_{1}, K_{1} \cdot d_{1}\right)$ and $\left(N_{2} \cdot K_{2} \cdot d_{2}\right)$, where $N_{i} . K_{i}$. and $d_{i}$ are codeword length, input information block length, and minimum hamming distance for the code $C_{i}$ respectively. The complete TPC matrix can be obtained by placing the $\left(K_{1} \times K_{2}\right)$ data in Array of $K_{2}$ rows and $K_{1}$ columns and encoded the $K_{2}$ rows using $C_{1}$ and finally encode the $N_{1}$ columns using $C_{2}$ to produce $N_{2}$, so the result will be TPC with $\left(K_{1} \times K_{2} . N_{1} \times\right.$ $N_{2} . d_{1} \times d_{2}$ ) as shown in Fig. 1. For this paper we assume the two component codes $C_{1}$. and $C_{2}$ are identical and have the same parameters $N_{1}=N_{2} \cdot K_{1}=K_{2}$. and $d_{1}=d_{2}$. In below paragraphs a summary of the steps adopted in this paper:

\section{A. Distributed Coded System}

To establish the distributed encoding for the TCP, we implement two scenarios the first with single relay and the second with double relays. In which relay nodes are located in the midway between source and destination. The source (S) broadcast the $(K \times N)$ codewords matrix encoded by $\mathrm{C}$ to the destination (D) and the relays (R) that are located in the midway between source and destination as shown in Fig. 2. Separations between Rs and D are as in [21] and [22], where the distance between $\mathrm{S}$ and $\mathrm{D}$ is normalized. The transmitted codeword $C=C_{1}, C_{2}, \cdots, C_{N}$ is modulated by Binary Phase Shift Keying BPSK to get $X=X_{1}, X_{2} \cdots X_{N}$. So the received signal at $\mathrm{R}$ and $\mathrm{D}$ in the first time slot are:

$r_{s d i}=\alpha_{s d i} h_{s d i} X_{i}+W_{s d i}$

$r_{s r i}=\alpha_{s r i} h_{s r i} X_{i}+W_{s r i}$

Where $X_{i} .1<i<N$ is the modulated transmitted horizontally codeword from source, and $h_{s d i}, h_{s r i}$ and $W_{s d i}$, $W_{s r i}$ are the fading coefficients and AWGN of S-D and S-R links respectively. And $\alpha_{s d i}, \alpha_{s r i}$ are the path loss attenuation for the two links affected by distance between nodes $\alpha_{s d i}=$ 1 , and $\alpha_{s r i}=(1-\lambda)^{-\mu}$ where $\mu$ is the path loss exponent and $\lambda$ is the distance between relay and destination. The received sequence at $\mathrm{R}$ is decoded by Chase $\Pi$ SISO decoder as in [23] and the detected information words $K_{1}$ are reencoded horizontally by component code $\mathrm{C}$ and encoded vertically to produce vertical parity part $V_{p}$ as shown in Figure 1. In the second time slot the coded vertically part $V_{p}$ is transmitted from $\mathrm{R}$ to $\mathrm{D}$ and received as:

$r_{r d i}=\alpha_{r d i} h_{r d i} X_{i}^{\prime}+W_{r d i}$

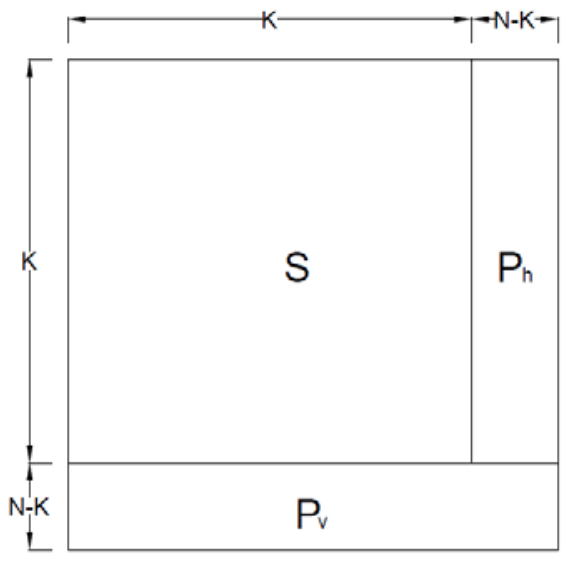

Fig. 1. The structure of TPC.

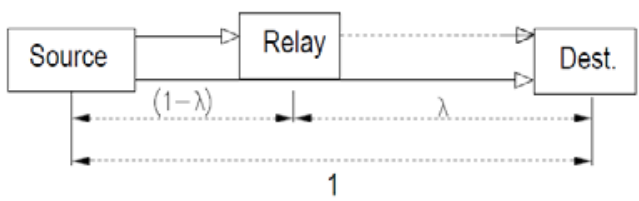

Fig. 2. Cooperative Model with Three Terminals.

Where $X^{\prime}{ }_{i}, 1<i<N$ is the modulated BPSK symbols of vertical parity column $V_{p}$, and $h_{r d i}, W_{r d i}$ are the fading coefficients and AWGN of R-D link. It should be noticed that the transmitted power from $\mathrm{S}$ and $\mathrm{R}$ are equal and path loss exponent $\mu$ is 2 . So we obtain the values of:

$S N R_{s r}=\frac{S N R_{S d}}{(1-\lambda)^{2}} \quad, S N R_{r d}=\frac{S N R_{S d}}{(\lambda)^{2}}$

From (4) the variance of $W_{s r}$ and $W_{r d}$ in (1,2 and 3) can be expressed as : $\sigma_{s r}^{2}=(1-\lambda)^{2} \sigma_{s d}^{2} \cdot \sigma_{r d}^{2}=(\lambda)^{2} \sigma_{s d}^{2}$ Where the variance of $W_{s d}=\sigma_{s d}^{2}, W_{r d}=\sigma_{r d}^{2}$, and $W_{s r}=\sigma_{s r}^{2}$.

\section{B. Distributed TPC Decoding using Single Relay}

The two parts of TPC matrix $\left\{\mathrm{S}, P_{h}\right\}$ and $\left\{P_{v}\right\}$ are received in $\mathrm{D}$ at the second time slot via direct link and relay channel with SNRs as in (1) and (3). At D the received $\left\{\mathrm{S}, P_{h}\right\}$ and $\left\{P_{v}\right\}$ are arranged to construct TPC as in Fig. 1. In this paper iterative chase pyndiah decoder as shown in Fig. 3 is used as SISO decoder to decode the received $\left\{P_{v}\right\}$ part from $\mathrm{R}$ by column decoder and received rows $\left\{\mathrm{S}, P_{h}\right\}$ through direct link by row decoder. Soft decoding for $\left\{P_{v}\right\}$ in columns decoder is performed using chase algorithm as following:

1- The hard decisions vector $R_{d}=\left(r_{d 1} \cdot r_{d 2} \cdot \cdots \cdot r_{d N}\right)$ is generated from the soft received sequence $r_{r d}=$ $r_{r d 1} \cdot r_{r d 2} \cdots \cdot r_{r d N}$ according to:

$r_{r d i}=\left\{\begin{array}{l}1 . \text { if } r_{r d i} \geq 0 \\ 0 . \text { other wise }\end{array}\right\}$

Where $i \in\{1.2 \ldots . N\}$

2- The reliability of $r_{r d i}$ component $\left|r_{r d i}\right|$ is ordered and positions of $\mathrm{P}$ of Least Reliable Bits LRB are determined, then $2^{P}$ test patterns $T_{l}$ are obtained by placing combinations of ones and zeros at P LRB and zeros in the remaining positions. 
3- $\quad$ The test sequence $Z_{l}$ is obtained by modul-2 addition between $T_{l}$ and $R_{d}$ as:

$Z_{l}=R_{d} \oplus T_{l}$

Where $l \in\left\{1.2 \ldots .2^{P}\right\}$

4- Syndrome is calculated for each $Z_{l}$ by $S_{l}=Z_{l} \cdot H^{T}$, where $l \in\left\{1.2 \ldots .2^{P}\right\}$ and $H^{T}$ is the transposed parity check matrix of the component code $C$. subsequently error correct based syndrome is conducted to generate valid codeword $C^{l}=\left(C_{1}^{l} . C_{2}^{l}, \ldots . C_{N}^{l}\right)$

5- Squared Euclidian Distance (SED) is calculated between $R_{d}$ and $C_{l}$ according to :

$\left|r_{d}-C^{l}\right|=\sum_{i=1}^{N}\left[r_{d i}-\left(2 C_{i}^{l}-1\right)\right]^{2}$

6- The best candidate word $\mathrm{D}$ is chosen by calculating the minimum SED among the $2^{P}$ candidate codewords by:

$D=\operatorname{argmin}\left(C^{l} \cdot\left|R_{d}-C^{l}\right|\right)$

7- In the present of competing codeword D related to the $i_{t h}$ bit position the extrinsic information is calculated for the column decoder as:

$w_{i C}=\frac{\left.\left\|r_{d}-C^{l(K)}\right\|^{2}-\|\left(r_{d}\right)-D\right) \|^{2}}{4}\left(2 d_{i}-1\right)-r_{i}$

Where $d_{i}$ is the $i_{t h}$ element decision codeword $\mathrm{D}$ and $C^{l(i)}$ is the competing codeword with minimum SED among the candidate codeword carrying value different from the $i_{t h}$ of $C^{l(K)}$. if this candidate codeword not exist $w_{i}$ is calculated as $w_{i}=\beta\left(2 d_{i}-1\right)$, where $\beta$ is the reliability factor and presented as in [9].

8- Once the extrinsic information is determined the input of next decoding stage (rows decoder) is inserted as follow:

$r_{i}^{\prime}=r_{s d i}+\alpha w_{i}$

Where $\alpha$ is the wight factor as in [1in parallel] to combat high standard deviation in extrinsic $w_{i}$ and high BER during first iteration. And $r_{s d i}$ is the received sequence from direct link which contain horizontally coded words $\left\{\mathrm{S}, P_{h}\right\}$.

All operations above are repeated on all bits of TPC codewords from step 1 to 8 to achieve the first half iteration as shown in Fig. 3 where the integer $m$ is the half iteration.

\section{Distributed TPC Decoding using Multiple Relays}

In this paper only two relays are considered for demonstration cooperative system with multiple relays. The S broadcast the block $\left\{\mathrm{S}, P_{h}\right\}$ to Rs and D. The two relays act independently, the two received sequence at Rs are decoded by chase $\Pi$ SISO decoder as in [23] and the detected information blocks are encoded again horizontally and vertically by component coded $\mathrm{C}$ to construct at TPC as shown in Fig. 1. In the second time slot the encoded vertical block $\left\{P_{v}\right\}$ are transmitted from both relays to destination as:

$r_{1 r d i}=\alpha_{r d 1 i} h_{r d 1 i} X_{r d 1 i}^{\prime}+W_{r d 1 i}$

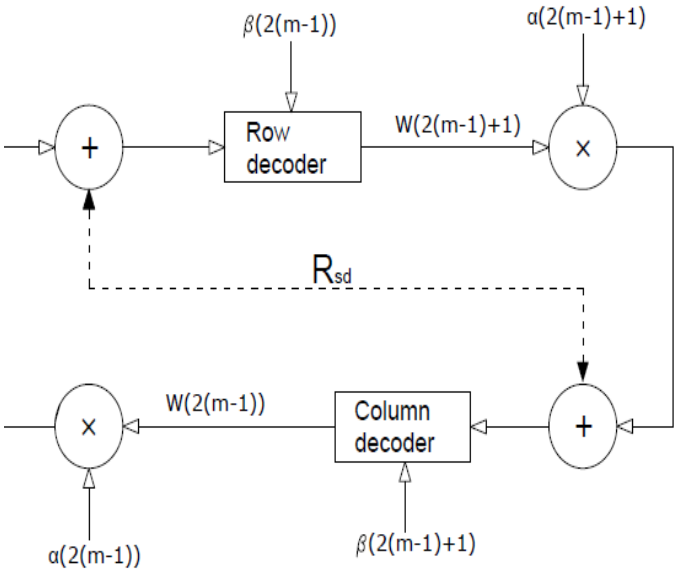

Fig. 3. Original Iterative Chase Pyndiah Decoder.

$r_{2 r d i}=\alpha_{r d 2 i} h_{r d 2 i} X_{r d 2 i}^{\prime}+W_{r d 2 i}$

Where $X_{r d 1 i}^{\prime}$ and $X_{r d 2 i}^{\prime}, 1 \leq i \leq N$ are the modulated vertically coded symbols from the two relays. $h_{r d 1 i}$ and $h_{r d 2 i}$ are the fading coefficients for the first and second relay links to destination respectively. The vertically parity coded parts are received from $\mathrm{R}$ and horizontally codewords from $\mathrm{S}$ through direct link are $\left\{P_{v 1} \cdot P_{v 2} \cdot P_{h} \cdot S\right\}$. For each generated vertical parities $\left\{P_{v 1} \cdot P_{v 2} \cdot\right\}$ there are two decoding stages at D: first through rows and then through columns decoder. So in this paper the proposed modified chase pyndiah decoder that contain multiple integrated sub stages for each iteration as shown in Fig. 4 is used. The first sub stage receives $r_{1 r d}$ sequence the contains vertical parties columns $\left\{P_{v 1}\right\}$ from the first relay are inserted to the first row decoder in sub stage 1 which perform the decoding process as explained in previous subsection for single relay case with steps from 1to 8. The main difference in the modified chase pyndiah decoder in this work is that the extrinsic of the column decoder $w_{i c}{ }^{\prime}$ in the first sub stage will be the input extrinsic for the next row decoder in the second sub stage and can be written as

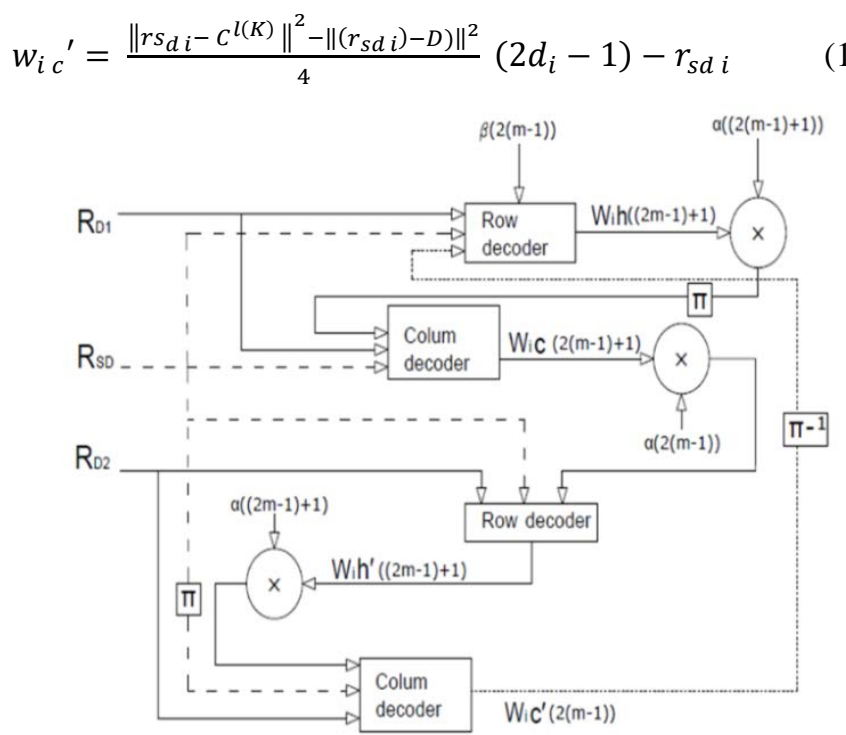

Fig. 4. Modified Iterative Chase Pyndiah Decoder. 
And the input sequence for the row decoder in the second sub stage will be:

$$
r 1^{\prime}=r_{2 r d i}+\alpha w_{i c}^{\prime}
$$

The rows decoder in the second sub stage will implement the decoding procedures for single relay case following steps from 1to 8. Extrinsic of row decoder of second sub stage $w_{i n}^{\prime}$ will be the input for columns decoder exactly as in first sub stage.

$r 2^{\prime}=r_{s d i}+\alpha w_{i h}^{\prime}$

\section{RESULTS AND DISCUSSION}

In this section the simulation results obtained for distributed TPC utilizing chase pyndiah decoder for single relay and multiple relays are shown. In this paper a squared $(15,11,3)$ hamming code based TPC with code length 225 is used. For simulation positions of $\mathrm{P}=10$ of Least Reliable Positions LRB are determined. The simulation is carried over AWGN so the block fading coefficients in (1), (2), and (3) are normalized to 1 . Also the relays are assumed to be centered at the distance between source and destination. The error correcting performances will be represented in terms of BER and Signal to Noise Ratio (SNR $=E_{b} / N_{0}$ ). The BER of TPC $(15.11 .3)^{2}$ code over an single link AWGN (un-cooperative) channel using chase pyndiah decoder at destination is shown in Fig. 5. It can be observed that the fifth iteration reaches $\mathrm{BER}=10^{-5}$ at $6 \mathrm{~dB}$ while reaches $\mathrm{BER}=10^{-3}$ at $4 \mathrm{~dB}$ with 3 $\mathrm{dB}$ gain over uncoded system. In Fig. 6, the BER the performance results are shown for the same TPC but over Rayleigh fading channel. The fifth iteration reaches BER= $10^{-3}$ at $15 \mathrm{~dB}$ with more than $6 \mathrm{~dB}$ gain compared to uncorded BPSK system.

\section{A. For Single Relay}

The simulation results for TPC coded system utilizing single relay in cooperative system for free space propagation and the path loss exponent $\mu=2$ over AWGN and Rayleigh fading channels utilizing distributed coding and decoding with iterative chase pyndiah decoder at destination are shown respectively in Fig. 7 and Fig. 8.

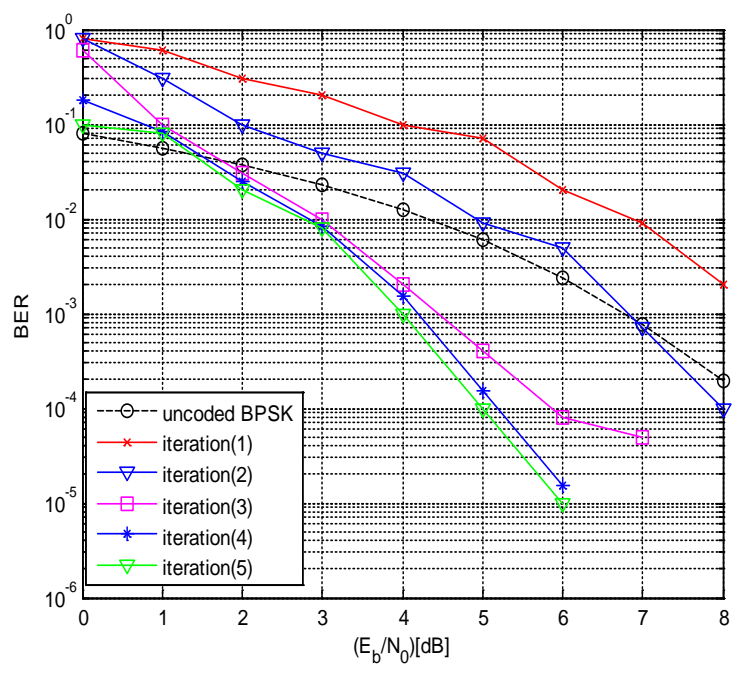

Fig. 5. The BER of the TPC over an AWGN Channel without Relays.

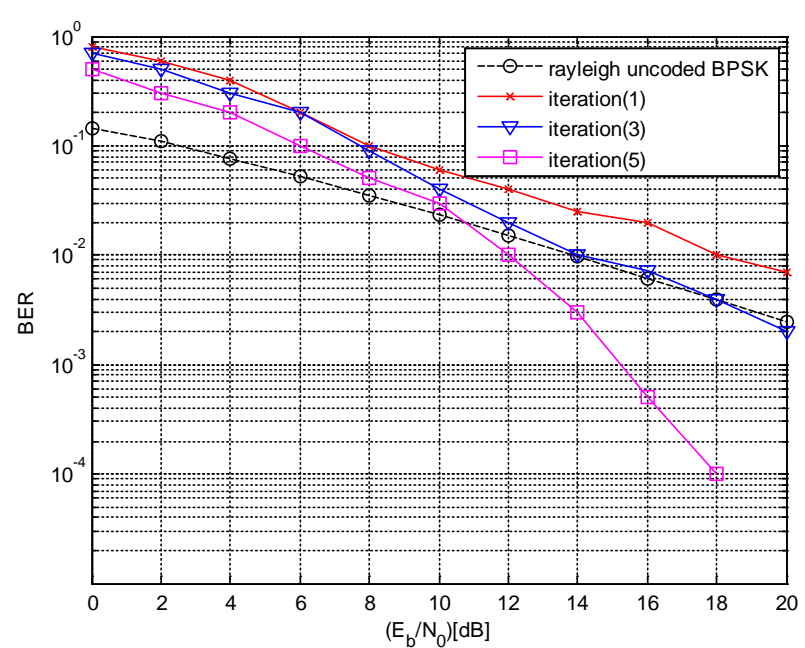

Fig. 6. The BER of the TPC over Rayleigh Fading Channel without Relays.

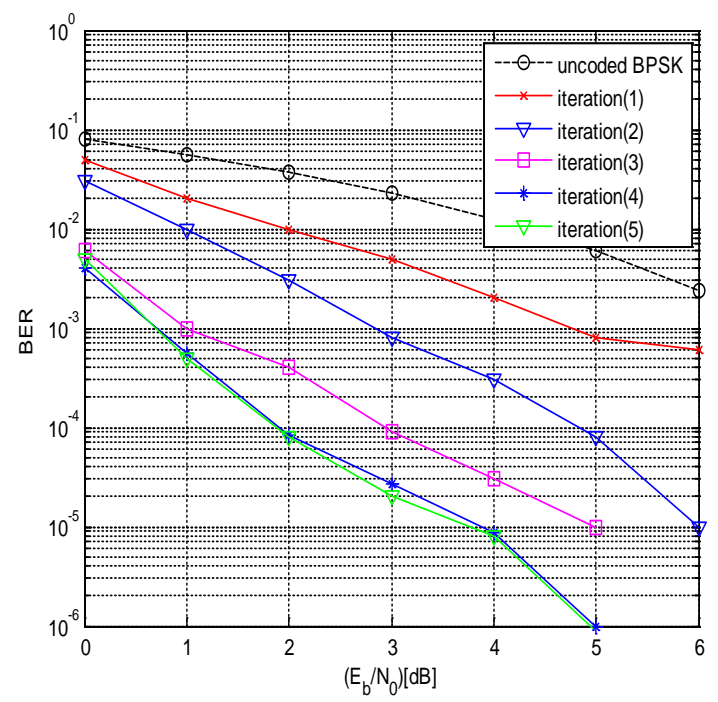

Fig. 7. BER Performance of Distributed TPC utilizing Single Relay over AWGN.

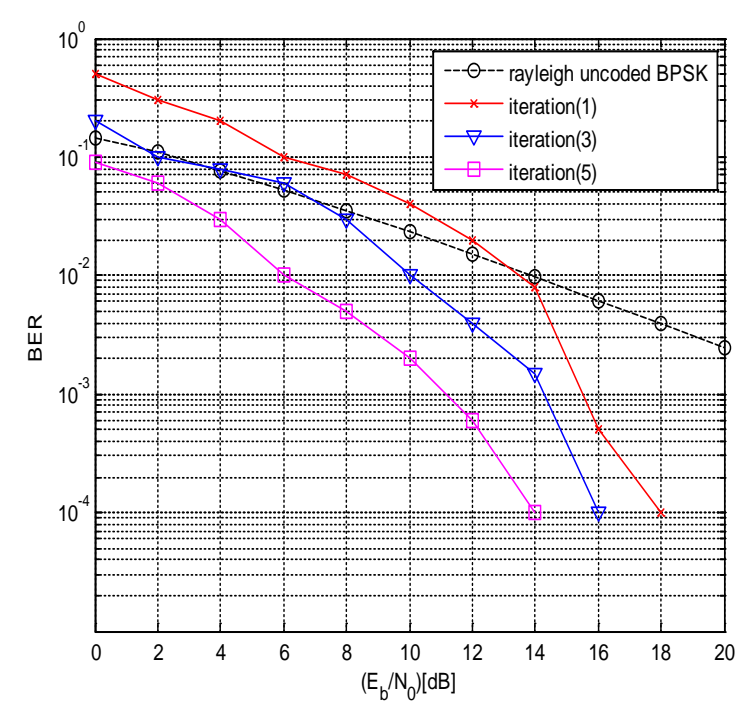

Fig. 8. BER Performance of Distributed TPC utilizing Single Relay over Rayleigh Fading Channel. 
The BER performance for distributed TPC utilizing single relay and iterative chase pyndiah over AWGN is shown in Fig. 7. The proposed distributed TPC decoder at destination with vertical columns parity $\left\{P_{v}\right\}$ from the relay is the input of rows decoder and the horizontally codewords from source \{ $\left.S . P_{h}\right\}$ are the input of columns decoder. The fifth Iteration reaches $10^{-5}$ BER at $S N R=4 \mathrm{~dB}$ with coding gain about $2 \mathrm{~dB}$ compared uncooperative TPC over AWGN in Fig. 5. In Fig. 8 the BER performance for distributed TPC utilizing single relay and iterative chase pyndiah over Rayleigh fading channel is shown. With same distributed decoding for TPC as in the system in Fig. 8 the fifth iteration reaches $10^{-4}$ BER at $14 \mathrm{~dB}$ SNR with coding gain about $4 \mathrm{~dB}$ compared to uncooperative case TPC in Fig. 6 for the same system parameters.

\section{B. For Multiple Relays}

In this paper only two relays are considered to construct the cooperative system. Where the proposed distributed TPC decoder at destination receives the vertical columns parity \{ $\left.P_{v 1}\right\}$ from the first relay as the input of row decoder and the horizontally codewords from source $\left\{S . P_{h}\right\}$ are the input of columns decoder in the first sub stage decoding. While receives the vertical columns parity $\left\{P_{v 2}\right\}$ from the second relay as the input of rows decoder and the horizontally codewords from source $\left\{S . P_{h}\right\}$ are the input of columns decoder in the second sub stage decoding. And the extrinsic of the columns decoder $w_{i c}{ }^{\prime}$ in the first sub stage will be the input extrinsic for the next row decoder in the second sub stage of the modified iterative chase pyndiah decoder.

We compare the performance of distributed TPC using modified iterative chase pyndiah decoder utilizing two relays with distributed original iterative chase pyndiah decoder utilizing one relay over AWGN and Rayleigh fading channel. In Fig. 9 the fifth iteration over AWGN reaches BER $=10^{-5}$ at SNR $=2.7 \mathrm{~dB}$ approximately while the distributed TPC iterative chase pyndiah with single relay at Fig. 7 reaches the same BER at SNR $=4 \mathrm{~dB}$ while the uncooperative reaches the same BER at $\mathrm{SNR}=6 \mathrm{~dB}$. So it can be observed that modified chase pyndiah decoder with 2 relays achieves $1.3 \mathrm{~dB}$ gain over single relay and $3.3 \mathrm{~dB}$ gain over un-cooperative system.

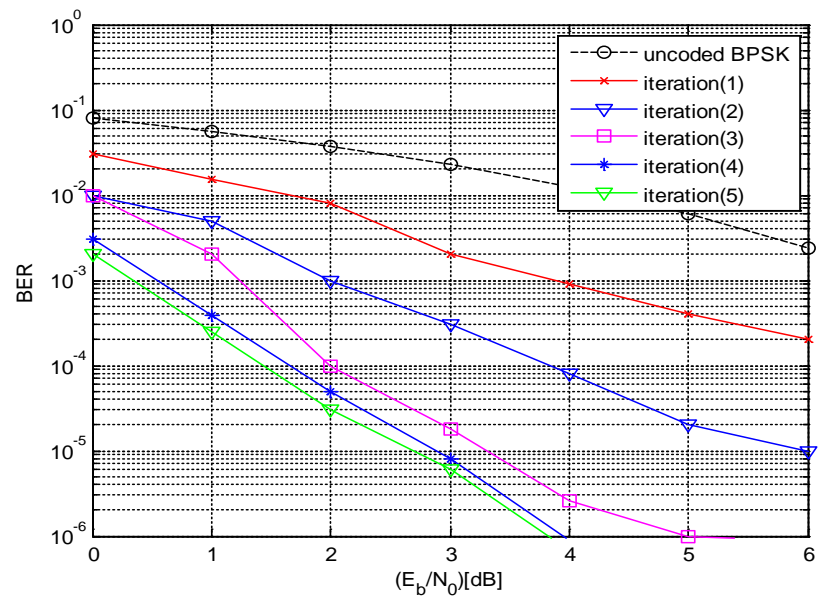

Fig. 9. BER Performance of Distributed TPC utilizing Multiple Relays over AWGN.

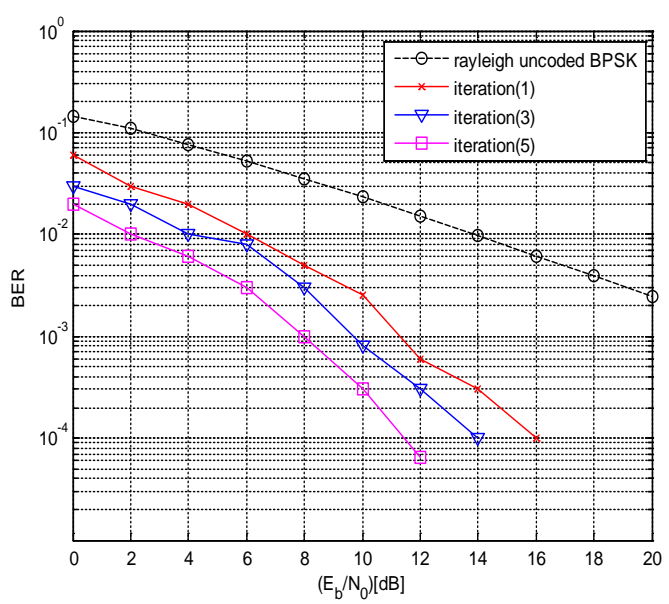

Fig. 10. BER Performance of Distributed TPC utilizing Multiple Relays over Rayleigh Fading Channel.

For Rayleigh Fading channel in Fig. 10 with same system parameters of Fig. 9 the distributed decoding with iterative modified chase pyndiah decoder shows coding gain of $3 \mathrm{~dB}$ for the fifth iteration compared single relay scenario at BER= $10^{-4}$ in Fig. 8 and here it can be noticed the efficiency of increasing number of iterations with proposed modified chase pyndiah iterative decoder.

\section{CONCLUSION}

The results presented in this paper illustrate the distributed coding and decoding of TPC using iterative chase pyndiah decoder over single and multiple relay networks. Two scenarios were investigated for distributed coding and decoding , the first utilizing one relay in cooperative network where the original chase pyndiah decoder at designation receives $\left\{P_{v}\right\}$ vertical parity codewords from relay as the input of rows decoder and the horizontally codewords $\left\{S . V_{h}\right\}$ from source as input to column decoder. In the second scenario multiple relays are utilized and multiple copies of vertical parity parts of TPC are sending to destination to be decoded by modified iterative chase pyndiah decoder with multiple integrated stages for each iteration. Simulation results for proposed system with single relay and multiple relays modified iterative chase Pyndiah decoder show gain improvement respectively over BER performance of uncooperative system.

\section{REFERENCES}

[1] Zhao and M. C. Valenti, "Distributed turbo coded diversity for relay channel," Electronics Letters, vol. 39, no. 10, pp. 786-787, 2003.

[2] M. C. Valenti and Bin Zhao, "Distributed turbo codes: towards the capacity of the relay channel," in IEEE 58th Vehicular Technology Conference, VTC, Orlando, 2003.

[3] K. Anwar and T. Matsumoto, "Accumulator-Assisted Distributed Turbo Codes for Relay Systems Exploiting Source-Relay Correlation," IEEE Communications Letters, vol. 16, no. 7, pp. 1114-1117, July 2012.

[4] Mughal, S.; Yang, F.-F.; Ejaz, S, " Asymmetric turbo code for coded cooperative wireless communication based on matched interleaver with channel estimation and multi-receive antennas at the destination.," Radio engineering, vol. 26, pp. 878-889, 2017.

[5] C.Zhao, F. Yang, R. Umar and S. Mughal, "Two-Source Asymmetric Turbo-Coded Cooperative Spatial Modulation Scheme with Code," electronics, vol. 9, no. 1, pp. 1-20, 2020. 
[6] C. Argon and S. W. McLaughlin, "An efficient Chase decoder for turbo product codes," IEEE Transactions on Communications, vol. 52, no. 6, pp. 896-898, June 2004.

[7] C. Xu, Y. Liang and W. S. Leon, "A Low Complexity Decoding Algorithm for Turbo Product Codes," in IEEE Radio and Wireless Symposium, Long Beach, CA, 2007.

[8] J. Cho and W. Sung, "Reduced complexity Chase-Pyndiah decoding algorithm for turbo product codes," in IEEE Workshop on Signal Processing Systems (SiPS), Beirut, 2011.

[9] R. M. Pyndiah, "Near-optimum decoding of product codes: block turbo codes," IEEE Transactions on Communications, vol. 46, no. 8, pp. 10031010, Aug 1998.

[10] S. Yoon, B. Ahn and J. Heo, "An advanced low-complexity decoding algorithm for turbo product codes based on the syndrome," EURASIP Journal on Wireless Communications and Networking, vol. 126, no. 16, pp. 1-31, 2020.

[11] A. Sendonaris, E. Erkip and B. Aazhang, "User cooperation diversity. Part I. System description," IEEE Transactions on Communications, vol. 51, no. 11, pp. 1927-1938, Nov.2003.

[12] H. Liang, A. Liu, J. Dai, J. Liu, and C. Gong, "Design and analysis of polar coded cooperation with incremental redundancy for IoT in fading channels," IET Communications, vol. 15, no. 4, pp. 595-602, 2021.

[13] R. Hu and J. Li, "Practical Compress-Forward in User Cooperation: Wyner-Ziv Cooperation," in IEEE International Symposium on Information Theory, Seattle, WA, 2006.

[14] H. H. Sneessens and L. Vandendorpe, "Soft Decode and Forward Improves Cooperative Communications," in 2005 6th IEE International Conference on 3G and Beyond, Washington, DC, 2005.
[15] Y. Li, B. Vucetic, T. F. Wong and M. Dohler, "Distributed Turbo Coding with Soft Information Relaying in Multihop Relay Networks," IEEE Journal on Selected Areas in Communications, vol. 24, no. 11, pp. 20402050, Nov. 2006.

[16] Y. Hairej, A. Darmawan and H. Morikawa, "Cooperative Diversity using Soft Decision and Distributed Decoding," in 2007 16th IST Mobile and Wireless Communications Summit, Budapes, 2007.

[17] E. A. Obiedat, G. Chen and L. Cao, "Distributed Turbo Product Codes over Multiple Relays," in 2010 7th IEEE Consumer Communications and Networking Conference, Las Vegas, 2010.

[18] E. A. Obiedat, L.Cao, "Cyclic Interleaving for Multiple Vertical Parities in Distributed Turbo Product Codes," Recent Patents on Signal Processing, vol. 4, no. 1, pp. 12-17, 2014.

[19] E. A. Obiedat and L. Cao, "Soft Information Relaying for Distributed Turbo Product Codes (SIR-DTPC)," IEEE Signal Processing Letters, vol. 17, no. 4, pp. 363-366, April. 2010.

[20] E. A. Obiedat and L. Cao, "Power Allocation for Distributed Turbo Product Codes (DTPC)," in IEEE Global Telecommunications Conference GLOBECOM 2010, Miami, FL, 2010.

[21] S. E. A. Alnawayseh and P. Loskot, "Cooperative Versus Receiver Coded Diversity with Low-Complexity Encoding and Decoding," in 2010 IEEE 71st Vehicular Technology Conference, Taipei, 2010.

[22] S.E. A. Alnawayseh, "Performance Comparison of Coded OFDM System with Cooperative Diversity and Multi- Antenna Receiver Diversity using QAM modulation," JJEE Jordan Journal of Electric Engineering, vol. 1, no. 2, 2015.

[23] D. Chase, "Class of algorithms for decoding block codes with channel measurement information," IEEE Transactions on Information Theory, vol. 18, no. 1, pp. 170-182, 1972. 